\title{
Variations
}

Variations

Revue internationale de théorie critique

22 | 2019

Gorz, l'intempestif

\section{Sortie du capitalisme, revenu d'existence et monnaies alternatives dans la pensée de Gorz}

\section{Christophe Fourel}

\section{(2) OpenEdition}

Journals

Édition électronique

URL : http://journals.openedition.org/variations/1194

DOI : 10.4000/variations. 1194

ISSN : 1968-3960

Éditeur

Les amis de Variations

Référence électronique

Christophe Fourel, «Sortie du capitalisme, revenu d'existence et monnaies alternatives dans la pensée de Gorz », Variations [En ligne], 22 | 2019, mis en ligne le 04 mars 2019, consulté le 01 mai 2019. URL : http://journals.openedition.org/variations/1194; DOI : 10.4000/variations.1194

Ce document a été généré automatiquement le 1 mai 2019.

Les ami•e•s de Variations 


\title{
Sortie du capitalisme, revenu d'existence et monnaies alternatives dans la pensée de Gorz
}

\author{
Christophe Fourel
}

1 Dans un entretien qu'André Gorz a accordé en 2005 (deux ans avant sa mort donc) à une revue brésilienne et publié à titre posthume dans le recueil Ecologica sous le titre «Richesse sans valeur, valeur sans richesse », il affirme ceci : «Je ne pense pas que le revenu d'existence puisse être introduit graduellement et pacifiquement par une réforme décidée "d'en haut". » Puis il ajoute un peu plus loin :

"Le revenu d'existence, quand il sera introduit, sera une monnaie différente de
celle que nous utilisons aujourd'hui. Elle n'aura pas les mêmes fonctions. Elle ne
pourra pas servir à des fins de domination, de puissance. Elle sera créée "d'en bas",
portée par une vague de fond, en même temps que des réseaux de coopératives
communales d'autoproduction [...] en réponse à une conjonction des différentes
formes de crise que nous sentons monter : crise climatique, crise écologique ; crise
de l'énergie et crise monétaire par suite de l'effondrement du système de crédit. ${ }^{1}$ "

2 Ces affirmations faites à la fin de sa vie sont importantes car en peu de lignes elles condensent à elles-seules les évolutions de la pensée d'André Gorz dans la dernière partie de son œuvre. Elles traduisent les options les plus radicales de sa pensée sur le rôle qu'il assigne au Revenu d'Existence. Elles méritent donc qu'on s'y attarde. Nous pouvons donc les prendre comme point de départ car elles nous permettent d'articuler notre réflexion en trois temps. D'abord revenir brièvement sur l'évolution de la pensée d'André Gorz quant à son soutien à l'idée d'instaurer un revenu d'existence. Ensuite explorer la question de la forme monnaie que pourrait prendre, selon Gorz, ce revenu d'existence. Enfin, nous terminons sur quelques considérations concernant les monnaies complémentaires telles qu'elles se développent aujourd'hui en France et en Europe et nous nous demandons si ces expériences peuvent nous aider à explorer les voies d'une sortie du capitalisme. 


\section{Gorz et le revenu d'existence}

3 On le sait, André Gorz a longtemps récusé la proposition du revenu d'existence. Parce qu'il y voyait un obstacle à son idée première : c'est le travail qu'il faut répartir de façon aussi égalitaire que possible, pour que nul ne vienne à en manquer et que l'ajustement se fasse par la réduction du temps travaillé par chacun. Puisque le travail qu'il appelait "hétéronome» est une nécessité sociale, mais que, en même temps, en limitant l'autonomie de ceux qui l'exercent, il va à l'encontre d'une vie bonne, autant faire que chacun porte sa part de fardeau. Une allocation universelle, estimait-il avant le milieu des années 1990, risquerait d'empêcher toute redistribution égalitaire du travail " hétéronome », donc de conforter les rapports sociaux de type capitaliste. C'est cette thèse qu'il a développé notamment à partir de 1983 lors de la parution de son ouvrage " Les Chemins du paradis », où il proposait l'instauration d'un « revenu social », un revenu déconnecté du volume de travail mais non du travail lui-même.

4 En 1997, dans Misères du présent, richesse du possible, il abandonna cette position suite aux échanges intellectuels fructueux qu'il aura eus en particulier avec les contributeurs et les contributrices de la revue Futur Antérieur (devenu Multitudes). Notamment parce que l'allocation universelle, en étant versée à tous sans aucune conditionnalité, allait inciter un nombre accru de personnes à préférer le développement d'activités bénévoles, artistiques, culturelles, familiales, d'entraide, etc., donc constituait un accélérateur de la transition vers un post-capitalisme et l'accroissement de l'autonomie des individus. En outre, ajoutait-il, dans l'économie de l'immatériel qui s'annonce, l'allocation universelle est mieux adaptée que la rémunération directe du travail (hétéronome) fourni par chacun, car l'efficacité productive relève désormais moins des efforts personnels que du niveau général des connaissances: le revenu doit donc être le plus socialisé possible, puisque l'efficacité productive s'appuie de plus en plus sur des connaissances socialisées. La vision gorzienne est sans nul doute celle d'une société où, peu à peu, la part de l'économique irait en déclinant, qu'il s'agisse du travail productif ou des revenus primaires, c'est-à-dire versés en contrepartie d'un apport productif. Cela étant, pour Gorz, l'allocation universelle d'un revenu d'existence ne peut devenir émancipatrice qu'à la condition qu'il soit :

5 Couplé à de véritables possibilités d'autoproduction ;

6 Véritablement inconditionnel car seule son inconditionnalité pourra préserver l'inconditionnalité des activités (accomplies pour elles-mêmes) ;

7 Suffisant car dans le cas contraire, elle aurait pour effet d'inciter à la recherche constante d'emploi de mauvaise qualité.

Soulignons pour finir cette partie qu'André Gorz ne considérait pas le revenu d'existence comme un but à atteindre mais comme un outil pour soutenir un processus de sortie du capitalisme (Gollain 2018).

\section{La forme monnaie du revenu d'existence}

9 Le revenu d'existence a depuis quelques années fait un retour en force dans le débat public. Sa promotion durant la campagne présidentielle en 2017 a largement contribué à ce que ce sujet sorte des discussions entre initiés pour venir se propager plus avant dans 
l'opinion. Malgré cette soudaine et notable notoriété, l'idée d'instaurer ce revenu d'existence n'a pas encore créé un véritable mouvement social qui puisse véritablement porter cette option. Plusieurs raisons peuvent l'expliquer. Une première est que le revenu d'existence a immédiatement été associé au renoncement de la valeur du travail et ses vertus intégratrices. Une deuxième, lié à la première, tient à son inconditionnalité, c'està-dire sans contrepartie et quel que soit le niveau de revenu par ailleurs. Une troisième relève de son financement. Sur ce dernier point, plusieurs solutions ont été proposées. Les deux principales (non exclusives l'une de l'autres d'ailleurs) consistent à substituer ce revenu à tout ou partie des transferts sociaux conditionnels actuellement en vigueur et/ ou à le financer pour partie par des impôts nouveaux, par exemple une taxe sur les transactions financières. Fondamentalement, il s'agit dans ces diverses modalités de financement, de réallouer du pouvoir d'achat déjà existant à travers une modification de la structure fiscale et social. C'est par exemple l'option privilégiée en 2015 par Marc de Basquiat et Gaspard Koenig en proposant le liber. Cependant, restreindre ainsi la question du financement du revenu d'existence à des enjeux fiscaux et budgétaires laisse entendre d'une part que l'on conçoit ce revenu comme le produit d'une nouvelle redistribution et d'autre part que l'on raisonne à masse monétaire donnée. Or, comme le laissent percevoir les deux citations d'André Gorz faites en introduction, ce sont deux sujets au travers desquels il a exprimé la plus grande radicalité de ses options vis-à-vis du revenu d'existence.

On sait que la quantité de monnaie en circulation dans l'économie ne dépend, en l'état actuel du système (qui fonctionne sur une monnaie quasi exclusivement issue du crédit bancaire), ni de notre sens de la justice sociale, ni de notre propension à assurer la soutenabilité environnementale de notre développement, ni même du soin que nous pourrions nous apporter mutuellement.

11 En réalité, une bonne partie des prétendues contraintes d'incitation jugées inhérentes au système économique est le résultat d'un choix politique : celui de déléguer à un secteur bancaire la fonction de créer l'intégralité des moyens de paiement que nous utilisons tous les jours.

12 Or, comme le souligne fort justement Christian Arnsperger, suivant ici André Gorz, «le problème est que le type de confiance sur lequel reposent la création et la circulation de la monnaie de dette bancaire dans nos économies n'est pas du tout le même que celui sur lequel reposerait le versement d'un revenu d'existence.» Dès lors, verser ce revenu d'existence en monnaie bancaire conventionnelle pose la question de la congruence entre les valeurs qu'entend incarner ce revenu et celles qu'incarne de facto la monnaie dans laquelle il sera versé.

13 C'est la raison pour laquelle, très tôt dans son œuvre (notamment depuis Les Chemins $d u$ paradis en 1983), André Gorz avait fait sienne l'idée de Jacques Duboin que le «revenu social» (comme il l'appelait à l'époque) soit un revenu de distribution et non pas un revenu de redistribution. Jacques Duboin avait en effet imaginé dans les années 1930 une économie de l'abondance, et non plus basée sur la rareté : l'économie distributive. Dans cette économie, les revenus distribués correspondraient à des parts de la richesse commune à tous au lieu de constituer la reconnaissance monétaire de l'effort de travail fourni par chacun.

14 Sous cet angle, le revenu d'existence et le type de monnaie dans lequel celui-ci serait versé ont partie lié dès lors qu'on veut promouvoir un système vecteur de justice sociale, de soutenabilité écologique et d'attention portée aux autres. 
Comme l'indique Christian Arnsperger, il ne s'agirait pas de supprimer la création monétaire par le crédit bancaire, mais de lui adjoindre d'autres mécanismes de création monétaire complémentaires venant contrebalancer les effets délétères de la monnaie conventionnelle et favoriser le maintien ou l'émergence d'échanges propices à une meilleure cohésion de la société.

De tels mécanismes sont précisément ceux que tentent de déployer les milliers d'initiatives de monnaies locales complémentaires qui ont vu le jour un partout dans le monde, notamment depuis la crise monétaire et financière de 2007-2008. Et que nous aborderons brièvement dans la troisième partie.

Pour conclure provisoirement, il n'est pas inutile de citer à nouveau André Gorz. C'est un extrait tiré de son livre L'Immatériel paru en 2003. Dans ce passage il envisage un retournement de la Bourse et les conséquences d'une dépression longue et profonde pour évoquer une solution :

«Seule une monnaie de consommation spécifique, différente de l'argent qui actuellement remplit quatre fonctions très différentes, créée et distribuée selon des critères politiques, non inflationniste par sa nature (péremption courte, circulation limitée), peut éviter l'implosion d'un système qui produit de plus en plus de marchandises en distribuant de moins en moins de moyens de paiement. Il est très vraisemblable qu'une telle monnaie sera introduite dans les vingt ans qui viennent, sous divers déguisements d'abord, avant d'oser s'appeler par son nom. "

Puis il ajoute en soulignant : «Selon les modalités et le contexte politique de son introduction, la monnaie de consommation sera ou ne sera pas le moyen grâce auquel le capitalisme survivra à sa propre mort en sauvant le règne de la marchandise et le pouvoir symbolique de l'argent. » En note de bas de page il prend le soin de préciser que cette idée a été pour la première fois imaginée par Jacques Duboin en 1930 en précisant que cette monnaie "n'a pas pour vocation de monétariser les échanges locaux réciproques ou les activités coopératives mais d'assurer l'accès de tous à ce qui ne peut être produit que sur la base d'une division macrosociale du travail ou à ce qui est productible sans travail, et donc sans valeur monétaire. »

\section{Les enseignements à tirer des expériences de monnaies locales complémentaires}

9 Venons-en maintenant aux monnaies alternatives. Pour l'illustrer, on peut d'abord partir d'une expérience qui s'est déroulé (et se déroule peut-être toujours) en Calabre. Certains villages de Calabre, dans le sud de l'Italie, retrouvent du dynamisme aujourd'hui grâce aux migrants... et aux monnaies locales. C'est le cas par exemple du village de Riace. Au cœur d'une région sinistrée, vidé de sa population par l'émigration déjà ancienne, où les services publics fermaient, ce village revit depuis 2010. On peut même parler d'une véritable renaissance, au point que le cinéaste allemand Wim Wenders y a réalisé un documentaire avec le soutien du Haut-Commissariat aux Réfugiés des Nations Unies en raison du caractère exemplaire de cette expérience.

20 Le coup de génie de Domenico Lucano, le maire de ce petit village de 1800 habitants, a été de parier sur l'accueil des réfugiés pour faire revivre sa commune et l'économie locale en associant les collectivités avoisinantes à son expérience et dans laquelle l'émission d'une monnaie locale a joué un rôle déterminant. 
21 Alors que les aides publiques tardaient à être versées à sa collectivité (celles de l'Etat et celles de l'UE pour permettre l'accueil des migrants), le maire a eu l'idée de créer une monnaie locale, gagée sur les fonds publics à venir, afin de verser directement les sommes dues et du pouvoir d'achat aux réfugiés installés en nombre dans sa commune. Les billets de cette nouvelle monnaie sont "frappés » à l'effigie des références politiques de Domenico Lucano : Gandhi, Martin Luther King, Che Guevara et Giuseppe Impastato (un journaliste italien assassiné par la mafia en 1978). Comme c'est toujours le cas pour les monnaies locales, l'utilisation de celle émise par la commune n'est utilisable que sur le territoire de Riace et ceux des autres communes associées à cette initiative. En très peu de temps, on a constaté une revitalisation de l'économie locale où cette monnaie est devenue une véritable «langue» commune pour tous les habitants du village alors que dans les rues résonnent désormais le kurdes, l'arabe, le swahili... sans oublier l'italien.

Avec le recul, les promoteurs de cette expérience n'hésitent pas à parler "d'effet multiplicateur " sur l'économie locale où les services publics ont rouvert (y compris l'école), où les maisons en ruine ont été réhabilitées, où l'artisanat local s'est développé (y compris et surtout celui produit par les migrants eux-mêmes). Ces mêmes promoteurs plaident donc désormais pour la solution de la répartition ciblée des réfugiés dans les zones où leur présence est susceptible d'avoir ce type d'effets positifs. Ils démontrent en outre que le financement de centre de détention serait quatre fois plus coûteux que la solution de l'émission de la monnaie locale expérimentée à Riace.

Ce petit exemple montre à lui seul une ébauche de ce que pourrait être une monnaie de distribution évoqué par André Gorz à la suite des thèses « abondancistes » développées par Jacques Duboin.

Comme le souligne fréquemment Bernard Lietaer, éminent spécialiste de la monnaie et des innovations monétaires, c'est que lorsqu'on évoque les risques graves auxquels est confrontée aujourd'hui l'humanité, on considère que la monnaie est extérieure au champ de toutes ces préoccupations. Or justement, il est fort probable que la monnaie, c'est-àdire la monnaie conventionnelle, basée sur l'émission via les dettes bancaires et contrôlée centralement, soit au cœur de bien des menaces auxquelles nous avons à affronter. Si cette hypothèse est juste, alors modifier la façon dont est créée et mise en circulation la monnaie pourrait figurer parmi les leviers nous permettant de trouver des solutions aux nombreux défis auxquels nous avons à faire face.

Il s'agirait alors de trouver des solutions aux problèmes que les monnaies conventionnelles se sont constamment montrés incapables de résoudre: le rétablissement des liens dans la communauté, la création de travail utile et gratifiant, une écologie durable, etc. Ce que Bernard Lietaer appelle «l'abondance durable» (Lietaer, 2012) : bref, une capacité de s'épanouir et de croître matériellement, émotionnellement et spirituellement sans gaspiller les ressources de nos descendants.

Il est vrai qu'il est difficile de percevoir comment certains mécanismes constitutifs du système monétaire en vigueur (celui tel qu'il fonctionne en dehors des périodes de crise) influencent les comportements individuels et collectifs. Bien sûr, certains de ces effets sont positifs: la monnaie moderne (conventionnelle) est par exemple à l'origine d'une profusion d'innovations scientifiques et industrielles sans précédent dans l'histoire. Mais en même temps, certains de ses mécanismes sont contre-productifs (au sens qu'Ivan Illich donnait à ce terme), comme si le système monétaire actuel contribuait à nuire à la monnaie elle-même. Sans entrer dans le détail, on peut au moins évoquer : l'amplification 
des fluctuations cycliques (instabilité du système monétaire fonctionnant sur des mécanismes autoréférentiels), la préférence pour la pensée à court terme, l'enfermement dans la croissance impérative, la concentration des richesses vecteur d'inégalité croissante et bien entendu la dévalorisation du capital social, source de cohésion sociale.

Nous vivons en effet avec la conviction que toutes les sociétés imposent (et doivent même imposer) le monopole d'une monnaie unique, émise par le pouvoir central et à laquelle s'attache un taux d'intérêt. En réalité, les historiens nous apprennent que des sociétés prospères ont encouragé la coexistence de différentes monnaies parallèles. Et que ce phénomène a souvent favorisé la stabilité économique, une meilleure répartition de la prospérité et une propension des acteurs économiques à tenir compte du long terme, plus que nous le faisons aujourd'hui.

Or, depuis plus d'une vingtaine d'années en effet, de nouvelles formes d'échange ont vu le jour en France. Ces manières renouvelées d'échanger ont d'abord pris la forme, dans les années 90, des systèmes d'échange local (SEL), inspirés des LETS anglo-saxons (Local Exchange Trading Systems). Prolongés et complétés, au début des années 2000, par l'apparition des Accorderies, formes de banques de temps initiées au Québec, puis importées en France sous l'impulsion décisive de la Fondation Macif. Enfin, il y a un peu plus de 5 ans sont nées les premières monnaies locales complémentaires (MLC) dont une quarantaine circule d'ores et déjà sur des territoires restreints.

29 Le développement de ces nouvelles formes d'échanges est un phénomène mondial qui s'est accéléré à la faveur des crises financières à répétition dont la plus importante est la crise des subprimes de 2008, suivie par celle des dettes souveraines en 2009-2010.

30 L'accélération et la diffusion de ces initiatives dans le double contexte de crise économique et de délitement du lien social comporte des enjeux essentiels à deux niveaux au moins :

31 Celui de dynamisation des territoires par la (re)localisation des activités, y compris (et peut-être surtout) en milieu rural.

32 Celui de nouvelles formes d'activités associant l'économique et le social dans le prolongement de celles qui ont conduit à l'épanouissement du secteur de l'économie sociale et solidaire.

Les MLC constituent ainsi des "dispositifs d'échange locaux de biens, de services, organisés autour d'une monnaie spécifique permettant à la fois d'évaluer et de régler des échanges " (Fourel, Magnen, Meunier 2015).

34 L'adjectif "complémentaire » souligne une articulation de ces monnaies au système monétaire en vigueur. Il est en effet important de préciser que pour la grande majorité de leurs initiateurs, ces nouveaux systèmes d'échanges n'ont pas vocation à remplacer la monnaie conventionnelle, mais à pallier les insuffisances et les effets délétères évoqués plus haut.

35 Reste que le tâtonnement expérimental auquel nous assistons actuellement autour des alternatives monétaires ne doit pas non plus être bridé dans sa dimension créatrice. Il est en effet important de laisser se déployer ces projets dans toutes leurs dimensions avant de les soumettre à un protocole d'évaluation nécessairement réducteur. À cet égard, il faut souligner que les expériences qui ont vu le jour autour des monnaies locales témoignent qu'on peut pousser très loin les frontières de l'innovation sociale. À notre connaissance, les monnaies complémentaires et les banques de temps sont en effet les rares expériences concrètes susceptibles d'inverser la hiérarchie des valeurs entre 
l'économique et le social, et pas seulement d'établir une bonne articulation entre ces valeurs. Dans le système capitaliste, le social est toujours second par rapport à l'économique. On produit et on valorise d'abord le social (bien souvent le social réparateur), vient ensuite par redistribution ou par une intervention d'action sociale plus ou moins publique. Or, à travers l'expérience d'innovation monétaire des MLC, il devient possible de faire remonter très en amont les valeurs de solidarité dans le processus de formation du revenu primaire. Le même raisonnement pourrait d'ailleurs être tenu s'agissant du respect des exigences environnementales.

Ainsi, au cœur même du capitalisme, une autre façon de concevoir l'échange tente à nouveau de se faire jour. Cette autre économie inverse le rapport entre la production de richesse marchande et la production de richesse humaine. La monnaie et son émission ne peuvent en effet rester extérieures à ces myriades d'innovations sociales qui se développent dans les domaines de l'économie solidaire, de l'économie participative ou encore de l'économie circulaire.

Les crises monétaires et financières à répétition, la dégradation des conditions d'existence et l'urgence des solutions à apporter au défi climatique nous forcent donc à concevoir d'autres façons de faire fonctionner l'économie en empruntant des voies allant dans le sens d'un «ré-encastrement » de celle-ci dans des projets guidés par des choix plus démocratiques et politiques. La sortie de la crise de civilisation dans laquelle nous sommes aujourd'hui enlisés ne passe pas par plus de marché ou par plus d'État. Elle doit, bien au contraire, être recherchée dans à la fois moins de marché, moins d'État et donc plus d'échanges qui ne soient commandés ni par l'argent pour son accumulation, ni par la réglementation administrative, mais fondés sur des réseaux de solidarité mutuelle. Telle était bien l'intuition d'André Gorz qui voyait dans le revenu d'existence un levier pour une transition vers une société postcapitaliste.

\section{BIBLIOGRAPHIE}

Arnsperger, Christian (2015), « Revenu de base, économie soutenable et alternatives monétaires ", L'Economie politique, $3^{\text {ème }}$ trimestre.

Clerc, Denis, Fourel, Christophe (2009), « André Gorz ou comment entrevoir le postcapitalisme », Esprit, novembre.

De Basquiat, Marc, Koenig, Gaspard (2015), Le Liber, un revenu de liberté pour tous, éditions de l'Onde.

Fourel, Christophe (2012), André Gorz, un penseur pour le XXIème siècle, La Découverte.

Fourel, Christophe (2017), « Le convivialisme et les monnaies complémentaires », in Reconstruction de la société. Analyses convivialistes, sous la direction de Marc Humbert.

Fourel, Christophe, Magnen, Jean-Philippe, Meunier, Nicolas (2015), D'autres monnaies pour une nouvelle prospérité, Le Bord de l'Eau.

Gorz, André (2008), Ecologica, Galilée. 
Gorz, André (2003), L’Immatériel, Galilée.

Gorz, André (1983), Les chemins du paradis, Galilée.

Lietaer, Bernard (2012), Halte à la toute-puissance des banques, Odile Jacob.

NOTES

1. Ecologica, 2008, p. 153-154

INDEX

Mots-clés : revenu d'existence, monnaies complémentaires, sortie du capitalisme

\section{AUTEUR}

\section{CHRISTOPHE FOUREL}

Christophe Fourel a dirigé les ouvrages suivants : André Gorz, un penseur pour le XXIème siècle (La Découverte, deuxième édition 2012) et Le Moment Gorz (Le Bord de l'Eau, 2017). 